\title{
Persistence Property and Asymptotic Description for DGH Equation with Strong Dissipation
}

\author{
Ke-chuang Wang \\ Department of Basic, Zhejiang Dongfang Vocational Technical College, Wenzhou, Zhejiang 325011, China \\ Correspondence should be addressed to Ke-chuang Wang; wangkechuang@126.com
}

Received 9 February 2013; Accepted 10 March 2013

Academic Editor: Yonghui Xia

Copyright (C) 2013 Ke-chuang Wang. This is an open access article distributed under the Creative Commons Attribution License, which permits unrestricted use, distribution, and reproduction in any medium, provided the original work is properly cited.

\begin{abstract}
The present work is mainly concerned with the Dullin-Gottwald-Holm (DGH) equation with strong dissipation. We establish a sufficient condition to guarantee global-in-time solutions, then present persistence property for the Cauchy problem, and describe the asymptotic behavior of solutions for compactly supported initial data.
\end{abstract}

\section{Introduction}

Dullin et al. [1] derived a new equation describing the unidirectional propagation of surface waves in a shallow water regime:

$$
\begin{aligned}
u_{t}- & \alpha^{2} u_{x x t}+c_{0} u_{x}+3 u u_{x}+\gamma u_{x x x} \\
& =\alpha^{2}\left(2 u_{x} u_{x x}+u u_{x x x}\right), \quad x \in \mathbb{R}, t>0,
\end{aligned}
$$

where the constants $\alpha^{2}$ and $\gamma / c_{0}$ are squares of length scales and the constant $c_{0}>0$ is the critical shallow water wave speed for undisturbed water at rest at spatial infinity. Since this equation is derived by Dullin, Gottwald, and Holm, in what follows, we call this new integrable shallow water equation (1) DGH equation.

If $\alpha=0$, (1) becomes the well-known $\mathrm{KdV}$ equation, whose solutions are global as long as the initial data is square integrable. This is proved by Bourgain [2]. If $\gamma=0$ and $\alpha=1$, (1) reduces to the Camassa-Holm equation which was derived physically by Camassa and Holm in [3] by approximating directly the Hamiltonian for Euler's equations in the shallow water regime, where $u(x, t)$ represents the free surface above a flat bottom. The properties about the well-posedness, blow-up, global existence, and propagation speed have already been studied in recent works [4-13], and the generalized version of a family of dispersive equations related to Camassa-Holm equation was discussed in [14].

It is very interesting that (1) preserves the bi-Hamiltonian structure and has the following two conserved quantities:

$$
\begin{gathered}
E(u)=\frac{1}{2} \int_{\mathbb{R}}\left(u^{2}+\alpha^{2} u_{x}^{2}\right) d x, \\
F(u)=\frac{1}{2} \int_{\mathbb{R}}\left(u^{3}+\alpha^{3} u u_{x}^{2}+c_{0} u^{2}-\gamma u_{x}^{2}\right) d x .
\end{gathered}
$$

Recently, in [15], local well-posedness of strong solutions to (1) was established by applying Kato's theory [16], and some sufficient conditions were found to guarantee finite time blow-up phenomenon. Moreover, Zhou [17] found the best constants for two convolution problems on the unit circle via variational method and applied the best constants on (1) to give some blow-up criteria. Later, Zhou and Guo improved the results and got some new criteria for wave breaking [18].

In general, it is quite difficult to avoid energy dissipation mechanism in the real world. Ghidaglia [19] studied the long time behavior of solutions to the weakly dissipative KdV equation as a finite dimensional dynamic system. Moreover, some results on blow-up criteria and the global existence condition for the weakly dissipative Camassa-Holm equation are presented in [20], and very related work can be found in 
$[21,22]$. In this work, we are interested in the following model, which can be viewed as the DGH equation with dissipation

$$
\begin{gathered}
u_{t}-\alpha^{2} u_{x x t}+c_{0} u_{x}+3 u u_{x}+\gamma u_{x x x}+\lambda\left(1-\alpha^{2} \partial_{x}^{2}\right) u \\
=\alpha^{2}\left(2 u_{x} u_{x x}+u u_{x x x}\right)
\end{gathered}
$$

where $x \in \mathbb{R}, t>0, \lambda\left(1-\alpha^{2} \partial_{x}^{2}\right) u$ is the weakly dissipative term and $\lambda$ is a positive dissipation parameter. Set $Q=(1-$ $\left.\alpha^{2} \partial_{x}^{2}\right)^{1 / 2}$, then the operator $Q^{-2}$ can be expressed by

$$
Q^{-2} f=G * f=\int_{\mathbb{R}} G(x-y) f(y) d y,
$$

for all $f \in L^{2}(\mathbb{R})$ with $G(x)=(1 / 2 \alpha) e^{-|x| / \alpha}$. With this in hand, we can rewrite (3) as a quasilinear equation of hyperbolic type

$$
\begin{aligned}
u_{t}+\left(u-\frac{\gamma}{\alpha^{2}}\right) u_{x}+\partial_{x} G \\
\quad *\left(u^{2}+\frac{\alpha^{2}}{2} u_{x}^{2}+\left(c_{0}+\frac{\gamma}{\alpha^{2}}\right) u\right)+\lambda u=0
\end{aligned}
$$

It is the dissipative term that causes the previous conserved quantities $E(u)$ and $F(u)$ to be no longer conserved for (3), and this model could also be regarded as a model of a type of a certain rate-dependent continuum material called a compressible second grade fluid [23]. Our consideration is based on this fact. Furthermore, we will show how the dissipation term affects the behavior of solutions in our forthcoming paper. As a whole, the current dissipation model is of great importance mathematically and physically, and it is worthy of being considered. In what follows, we assume that $c_{0}+\gamma / \alpha^{2}=0$ and $\alpha>0$ just for simplicity. Since $u(x, t)$ is bounded by its $H^{1}$-norm, a general case with $c_{0}+\gamma / \alpha^{2} \neq 0$ does not change our results essentially, but it would lead to unnecessary technical complications. So the above equation is reduced to a simpler form as follows:

$$
u_{t}+\left(u+c_{0}\right) u_{x}+\partial_{x} G * F(u)+\lambda u=0, \quad x \in \mathbb{R}, t>0,
$$

where

$$
F(u)=u^{2}+\frac{\alpha^{2}}{2} u_{x}^{2}
$$

The rest of this paper is organized as follows. In Section 2, we list the local well-posedness theorem for (6) with initial datum $u_{0} \in H^{s}, s>3 / 2$ and collect some auxiliary results. In Section 3, we establish the condition for global existence in view of the initial potential. Persistence properties of the strong solutions are explored in Section 4. Finally, in Section 5, we give a detailed description of the corresponding solution with compactly supported initial data.

\section{Preliminaries}

In this section, we make some preparations for our consideration. Firstly, the local well-posedness of the Cauchy problem of (6) with initial data $u_{0} \in H^{s}$ with $s>3 / 2$ can be obtained by applying Kato's theorem [16]. More precisely, we have the following local well-posedness result.

Theorem 1. Given that $u_{0}(x) \in H^{s}, s>3 / 2$, there exist $T=$ $T\left(\lambda,\left\|u_{0}\right\|_{H^{s}}\right)>0$ and a unique solution $u$ to $(6)$, such that

$$
u=u\left(\cdot, u_{0}\right) \in C\left([0, T) ; H^{s}\right) \cap C^{1}\left([0, T) ; H^{s-1}\right) .
$$

Moreover, the solution depends continuously on the initial data; that is, the mapping $u_{0} \rightarrow u\left(\cdot, u_{0}\right): H^{s} \rightarrow C([0$, $\left.T) ; H^{s}\right) \cap C^{1}\left([0, T) ; H^{s-1}\right)$ is continuous, and the maximal time of existence $T>0$ is independent of $s$.

Proof. Set $A(u)=\left(u+c_{0}\right) \partial_{x}, f(u)=-\partial_{x}\left(1-\alpha^{2} \partial_{x}^{2}\right)^{-1} F(u)-$ $\lambda u, Y=H^{s}, X=H^{s-1}, s>3 / 2$, and $Q=\left(1-\alpha^{2} \partial_{x}^{2}\right)^{1 / 2}$. Applying Kato's theory for abstract quasilinear evolution equation of hyperbolic type, we can obtain the local wellposedness of (6) in $H^{s}, s>3 / 2$ and $u \in C\left([0, T) ; H^{s}\right) \cap$ $C^{1}\left([0, T) ; H^{s-1}\right)$.

The maximal value of $T$ in Theorem 1 is called the lifespan of the solution in general. If $T<\infty$, that is $\lim \sup _{t \rightarrow T}\|u(\cdot, t)\|_{H^{s}}=\infty$, we say that the solution blows up in finite time, otherwise, the solution exists globally in time. Next, we show that the solution blows up if and only if its first-order derivative blows up.

Lemma 2. Given that $u_{0} \in H^{s}, s>3 / 2$, the solution $u=$ $u\left(\cdot, u_{0}\right)$ of (3) blows up in finite time $T<+\infty$ if and only if

$$
\liminf _{t \rightarrow T}\left\{\inf _{x \in \mathbb{R}}\left[u_{x}(x, t)\right]\right\}=-\infty .
$$

Proof. We first assume that $u_{0} \in H^{s}$ for some $s \in \mathbb{N}, s \geq 4$. Equation (6) can be written into the following form in terms of $y=\left(1-\alpha^{2} \partial_{x}^{2}\right) u$

$$
\begin{array}{r}
y_{t}+(y u)_{x}+\frac{1}{2}\left(u^{2}-\alpha^{2} u_{x}^{2}\right)_{x}+c_{0} y_{x}+\lambda y=0, \\
x \in \mathbb{R}, \quad t>0 .
\end{array}
$$

Multiplying (10) by $y=\left(1-\alpha^{2} \partial_{x}^{2}\right) u$, and integrating by parts, we have

$$
\begin{aligned}
\frac{d}{d t} \int_{\mathbb{R}} y^{2} d x & =2 \int_{\mathbb{R}} y y_{t} d x \\
& =-3 \int_{\mathbb{R}} u_{x} y^{2} d x-2 \lambda \int_{\mathbb{R}} y^{2} d x
\end{aligned}
$$

Differentiating (10) with respect to the spatial variable $x$, then multiplying by $y_{x}=\left(1-\alpha^{2} \partial_{x}^{2}\right) u_{x}$, and integrating by parts again, we obtain

$$
\begin{aligned}
\frac{d}{d t} \int_{\mathbb{R}} y_{x}^{2} d x= & 2 \int_{\mathbb{R}} y_{x} y_{x t} d x=-5 \int_{\mathbb{R}} u_{x} y_{x}^{2} d x \\
& +\frac{2}{\alpha^{2}} \int_{\mathbb{R}} u_{x} y^{2} d x-2 \lambda \int_{\mathbb{R}} y_{x}^{2} d x .
\end{aligned}
$$


Summarizing (11) and (12), we obtain

$$
\begin{aligned}
\frac{d}{d t}\left(\int_{\mathbb{R}}\left(y^{2}+y_{x}^{2}\right) d x\right) \\
=-\left(3-\frac{2}{\alpha^{2}}\right) \int_{\mathbb{R}} u_{x} y^{2} d x \\
\quad-5 \int_{\mathbb{R}} u_{x} y_{x}^{2} d x-2 \lambda\left(\int_{\mathbb{R}}\left(y^{2}+y_{x}^{2}\right) d x\right) .
\end{aligned}
$$

If $u_{x}$ is bounded from below on $[0, T)$, for example, $u_{x} \geq-C$, $C$ is a positive constant, then we get by (13) and Gronwall's inequality the following:

$$
\|y\|_{H^{1}}^{2} \leq \exp \{(K C-2 \lambda) t\}\left\|y_{0}\right\|_{H^{1}}^{2}
$$

where $K=\max \left\{5,\left(3-2 / \alpha^{2}\right)\right\}$. Therefore, the $H^{3}$-norm of the solution to (10) does not blow up in finite time. Furthermore, similar argument shows that the $H^{k}$-norm with $k \geq 4$ does not blow up either in finite time. Consequently, this theorem can be proved by Theorem 1 and simple density argument for all $s>3 / 2$.

Lemma 3. Let $u_{0} \in H_{\alpha}^{1}$, then as long as the solution $u(x, t)$ given by Theorem 1 exists, for any $t \in[0, T)$, one has

$$
\|u\|_{H_{\alpha}^{1}}^{2}=\exp (-2 \lambda t)\left\|u_{0}\right\|_{H_{\alpha}^{1}}^{2}
$$

where the norm is defined as

$$
\|u\|_{H_{\alpha}^{1}}^{2}=\int_{\mathbb{R}}\left(u^{2}+\alpha^{2} u_{x}^{2}\right) d x
$$

Proof. Multiplying both sides of (10) by $u$ and integrating by parts on $\mathbb{R}$, we get

$$
\begin{gathered}
\int_{\mathbb{R}} u y_{t} d x+\int_{\mathbb{R}}(y u)_{x} u d x+\int_{\mathbb{R}} \frac{1}{2}\left(u^{2}-\alpha^{2} u_{x}^{2}\right)_{x} u d x \\
+\int_{\mathbb{R}} c_{0} y_{x} u d x+\int_{\mathbb{R}} \lambda y u d x=0 .
\end{gathered}
$$

Note that

$$
\begin{gathered}
\int_{\mathbb{R}}(y u)_{x} u d x+\int_{\mathbb{R}} \frac{1}{2}\left(u^{2}-\alpha^{2} u_{x}^{2}\right)_{x} u d x=0, \\
\int_{\mathbb{R}} c_{0} y_{x} u d x=0 .
\end{gathered}
$$

Then, we have

$$
\int_{\mathbb{R}} u\left(u_{t}-\alpha^{2} u_{x x t}\right) d x+\int_{\mathbb{R}} \lambda\left(u^{2}-\alpha^{2} u u_{x x}\right) d x=0 .
$$

Hence,

$$
\begin{gathered}
\int_{\mathbb{R}} u u_{t} d x-\alpha^{2} \int_{\mathbb{R}} u u_{x x t} d x+\lambda \int_{\mathbb{R}} u^{2} d x \\
-\lambda \alpha^{2} \int_{\mathbb{R}} u u_{x x} d x=0 .
\end{gathered}
$$

Thus, we easily get

$$
\int_{\mathbb{R}}\left(u u_{t}+\alpha^{2} u_{x} u_{x t}\right) d x+\lambda \int_{\mathbb{R}}\left(u^{2}+\alpha^{2} u_{x}^{2}\right) d x=0,
$$

and, therefore,

$$
\frac{d}{d t}\|u\|_{H_{\alpha}^{1}}^{2}+2 \lambda\|u\|_{H_{\alpha}^{1}}^{2}=0 .
$$

By integration from 0 to $t$, we get

$$
\|u\|_{H_{\alpha}^{1}}^{2}=\exp (-2 \lambda t)\left\|u_{0}\right\|_{H_{\alpha}^{1}}^{2}, \quad \text { for any } t \in[0, \mathrm{~T}) \text {. }
$$

Hence, the lemma is proved.

We also need to introduce the standard particle trajectory method for later use. Consider now the following initial value problem as follows:

$$
\begin{gathered}
q_{t}=u(t, q)+c_{0}, \quad t \in[0, T), \\
q(0, x)=x, \quad x \in \mathbb{R},
\end{gathered}
$$

where $u \in C^{1}\left([0, T), H^{s-1}\right)$ is the solution to (6) with initial data $u_{0} \in H^{s},(s>3 / 2)$ and $T>0$ is the maximal time of existence. By direct computation, we have

$$
q_{t x}(t, x)=u_{x}(t, q(t, x)) q_{x}(t, x) .
$$

Then,

$$
q_{x}(t, x)=\exp \left(\int_{0}^{t} u_{x}(\tau, q(\tau, x)) d \tau\right)>0, \quad t>0, x \in \mathbb{R},
$$

which means that $q(t, \cdot): \mathbb{R} \rightarrow \mathbb{R}$ is a diffeomorphism of the line for every $t \in[0, T)$. Consequently, the $L^{\infty}$-norm of any function $v(t, \cdot)$ is preserved under the family of the diffeomorphism $q(t, \cdot)$, that is,

$$
\|v(t, \cdot)\|_{L^{\infty}}=\|v(t, q(t, \cdot))\|_{L^{\infty}}, \quad t \in[0, T) .
$$

Similarly,

$$
\begin{array}{ll}
\inf _{x \in \mathbb{R}} v(t, x)=\inf _{x \in \mathbb{R}} v(t, q(t, x)), & t \in[0, T), \\
\sup _{x \in \mathbb{R}} v(t, x)=\sup _{x \in \mathbb{R}} v(t, q(t, x)), & t \in[0, T) .
\end{array}
$$

Moreover, one can verify the following important identity for the strong solution in its lifespan:

$$
\frac{d}{d t}\left(y(q(x, t), t) q_{x}^{2}(x, t)\right)=-\lambda y(q(x, t), t) q_{x}^{2}(x, t) .
$$

We get that

$$
y(q(x, t), t) q_{x}^{2}(x, t)=y_{0}(x) \exp (-\lambda t)
$$

where $y(x, t)$ is defined by $y(x, t)=\left(1-\alpha^{2} \partial_{x}^{2}\right) u(x, t)$, for $t \geq 0$ in its lifespan. 
From the expression of $u(x, t)$ in terms of $y(x, t)$, for all $t \in[0, T), x \in \mathbb{R}$, we can rewrite $u(x, t)$ and $u_{x}(x, t)$ as follows:

$$
\begin{aligned}
u(x, t)= & \frac{1}{2 \alpha} e^{-x / \alpha} \int_{-\infty}^{x} e^{\xi / \alpha} y(\xi, t) d \xi \\
& +\frac{1}{2 \alpha} e^{x / \alpha} \int_{x}^{\infty} e^{-\xi / \alpha} y(\xi, t) d \xi
\end{aligned}
$$

from which we get that

$$
\begin{aligned}
u_{x}(x, t)= & -\frac{1}{2 \alpha^{2}} e^{-x / \alpha} \int_{-\infty}^{x} e^{\xi / \alpha} y(\xi, t) d \xi \\
& +\frac{1}{2 \alpha^{2}} e^{x / \alpha} \int_{x}^{\infty} e^{-\xi / \alpha} y(\xi, t) d \xi
\end{aligned}
$$

\section{Global Existence}

It is shown that it is the sign of initial potential not the size of it that can guarantee the global existence of strong solutions.

Theorem 4. Assume that $u_{0} \in H^{s}, s>3 / 2$, and $y_{0}=u_{0}-$ $\alpha^{2} u_{0 x x}$ satisfies

$$
\begin{aligned}
& y_{0}(x) \leq 0, \quad x \in\left(-\infty, x_{0}\right), \\
& y_{0}(x) \geq 0, \quad x \in\left(x_{0}, \infty\right),
\end{aligned}
$$

for some point $x_{0} \in \mathbb{R}$. Then, the solution $u(x, t)$ to $(6)$ exists globally in time.

Proof. From the hypothesis and (30), we obtain that $y(x, t) \geq$ $0, q\left(x_{0}, t\right) \leq x<\infty ; y(x, t) \leq 0,-\infty<x \leq q\left(x_{0}, t\right)$. According to (31) and (32), one can get that when $x>x_{0}$,

$$
\begin{aligned}
& u(q(x, t), t)+\alpha u_{x}(q(x, t), t) \\
& =\frac{1}{\alpha} e^{q(x, t) / \alpha} \int_{q(x, t)}^{\infty} e^{-\xi / \alpha} y(\xi, t) d \xi \geq 0,
\end{aligned}
$$

it follows that

$$
\begin{aligned}
-\alpha u_{x}(q(x, t), t) & \leq u(q(x, t), t) \leq\|u\|_{L^{\infty}} \\
& \leq \frac{\exp (-\lambda t)}{\sqrt{2 \alpha}}\left\|u_{0}\right\|_{H_{\alpha}^{1}} \leq \frac{1}{\sqrt{2 \alpha}}\left\|u_{0}\right\|_{H_{\alpha}^{1}},
\end{aligned}
$$

that is, $u_{x}(x, t)$ is bounded below. Similarly, when $x<x_{0}$,

$$
\begin{aligned}
& u(q(x, t), t)-\alpha u_{x}(q(x, t), t) \\
& =\frac{1}{\alpha} e^{-q(x, t) / \alpha} \int_{-\infty}^{q(x, t)} e^{\xi / \alpha} y(\xi, t) d \xi \leq 0,
\end{aligned}
$$

so $-\alpha u_{x}(q(x, t), t) \leq-u(q(x, t), t)$. We also get the bounded below result as above. Therefore, the theorem is proved by Lemma 2.

Corollary 5. Assume that $u_{0} \in H^{s}, s>3 / 2$, and $y_{0}=u_{0}-$ $\alpha^{2} u_{0 x x}$ is of one sign, then the corresponding solution $u(x, t)$ to (6) exists globally.

In fact, if $x_{0}$ is regarded as $\pm \infty$, we prove this corollary immediately from Theorem 4 .

\section{Persistence Properties}

In this section, we will investigate the following property for the strong solutions to (6) in $L^{\infty}$-space which behave algebraically at infinity as their initial profiles do. The main idea comes from the recent work of Himonas and his collaborators [7].

Theorem 6. Assume that for some $T>0$ and $s>3 / 2, u \in$ $C\left([0, T] ; H^{s}\right)$ is a strong solution of the initial value problem associated to $(6)$, and that $u_{0}(x)=u(x, 0)$ satisfies

$$
\left|u_{0}(x)\right|, \quad\left|u_{0 x}(x)\right| \sim O\left(x^{-\theta / \alpha}\right) \quad x \uparrow \infty,
$$

for some $\theta \in(0,1)$ and $\alpha \geq 1$. Then,

$$
|u(x, t)|, \quad\left|u_{x}(x, t)\right| \sim O\left(x^{-\theta / \alpha}\right) \quad x \uparrow \infty,
$$

uniformly in the time interval $[0, T]$.

Proof. The proof is organized as follows. Firstly, we will estimate $\|u(x, t)\|_{L^{\infty}}$ and $\left\|u_{x}(x, t)\right\|_{L^{\infty}}$. Then, we apply the weight function to obtain the desired result. In the following proof, we denote some constants by $c$; they may be different from instance to instance, changing even within the same line.

Multiplying (6) by $u^{2 n-1}$ with $n \in \mathbb{Z}^{+}$, then integrating both sides with respect to $x$ variable, we can get

$$
\begin{aligned}
& \int_{\mathbb{R}} u^{2 n-1} u_{t} d x+\int_{\mathbb{R}} u^{2 n-1}\left(u+c_{0}\right) u_{x} d x \\
& \quad+\int_{\mathbb{R}} u^{2 n-1} \partial_{x} G * F(u) d x=-\lambda \int_{\mathbb{R}} u^{2 n} d x .
\end{aligned}
$$

The first term of the above identity is

$$
\begin{aligned}
\int_{\mathbb{R}} u^{2 n-1} u_{t} d x & =\frac{1}{2 n} \frac{d}{d t}\|u(t)\|_{L^{2 n}}^{2 n} \\
& =\|u(t)\|_{L^{2 n}}^{2 n-1} \frac{d}{d t}\|u(t)\|_{L^{2 n}},
\end{aligned}
$$

and the estimates of the second term is

$$
\begin{gathered}
\int_{\mathbb{R}} u^{2 n-1} u u_{x} d x \leq\left\|u_{x}(t)\right\|_{L^{\infty}}\|u(t)\|_{L^{2 n}}^{2 n}, \\
c_{0} \int_{\mathbb{R}} u^{2 n-1} u_{x} d x=c_{0} \int_{\mathbb{R}}\left(\frac{u^{2 n}}{2 n}\right)_{x} d x=0 .
\end{gathered}
$$

In view of Hölder's inequality, we can obtain the following estimate for the third term in (39)

$$
\left|\int_{\mathbb{R}} u^{2 n-1} \partial_{x} G * F(u) d x\right| \leq\|u(t)\|_{L^{2 n}}^{2 n-1}\left\|\partial_{x} G * F(u)\right\|_{L^{2 n}}
$$

For the last term

$$
\left|\int_{\mathbb{R}} u^{2 n-1} \lambda u d x\right| \leq \lambda\|u(t)\|_{L^{2 n}}^{2 n}
$$


putting all the inequalities above into (39) yields

$$
\begin{aligned}
\frac{d}{d t}\|u(t)\|_{L^{2 n}} \leq & \left(\left\|u_{x}(t)\right\|_{L^{2 n}}+\lambda\right)\|u(t)\|_{L^{2 n}} \\
& +\left\|\partial_{x} G * F(u)\right\|_{L^{2 n}} .
\end{aligned}
$$

Using the Sobolev embedding theorem, there exists a constant

$$
M=\sup _{t \in[0, T]}\|u(x, t)\|_{H^{s}}
$$

such that we have by applying Gronwall's inequality

$$
\|u(t)\|_{L^{2 n}} \leq c e^{M t}\left(\|u(0)\|_{L^{2 n}}+\int_{0}^{t}\left\|\partial_{x} G * F(u)\right\|_{L^{2 n}} d \tau\right) .
$$

For any $f \in L^{1}(\mathbb{R}) \cap L^{\infty}(\mathbb{R})$, we know that

$$
\lim _{q \uparrow \infty}\|f\|_{L^{q}}=\|f\|_{L^{\infty}}
$$

Taking the limits in (46) (notice that $G \in L^{1}$ and $F(u) \in L^{1} \cap$ $L^{\infty}$ ) from (47), we get

$$
\|u(t)\|_{L^{\infty}} \leq c e^{M t}\left(\|u(0)\|_{L^{\infty}}+\int_{0}^{t}\left\|\partial_{x} G * F(u)\right\|_{L^{\infty}} d \tau\right) .
$$

Then, differentiating (6) with respect to variable $x$ produces the following equation:

$$
u_{x t}+u u_{x x}+c_{0} u_{x x}+u_{x}^{2}+\partial_{x}^{2} G * F(u)+\lambda u_{x}=0
$$

Again, multiplying (49) by $u_{x}^{2 n-1}$ with $n \in \mathbb{Z}^{+}$, integrating the result in $x$ variable, and considering the second term and the third term in the above identity with integration by parts, one gets

$$
\begin{aligned}
\int_{\mathbb{R}} u u_{x x} u_{x}^{2 n-1} d x & =\int_{\mathbb{R}} u\left(\frac{u_{x}^{2 n-1}}{2 n}\right)_{x} d x \\
& =-\frac{1}{2 n} \int_{\mathbb{R}} u_{x} u_{x}^{2 n} d x, \\
c_{0} \int_{\mathbb{R}} u_{x x} u_{x}^{2 n-1} d x & =c_{0} \int_{\mathbb{R}}\left(\frac{u_{x}^{2 n-1}}{2 n}\right)_{x} d x=0,
\end{aligned}
$$

so, we have

$$
\begin{aligned}
\int_{\mathbb{R}} u_{x t} u_{x}^{2 n-1} d x-\frac{1}{2 n} \int_{\mathbb{R}} u_{x} u_{x}^{2 n} d x+\int_{\mathbb{R}} u_{x}^{2 n+1} d x \\
=-\int_{\mathbb{R}} u_{x}^{2 n-1} \partial_{x}^{2} G * F(u) d x-\lambda \int_{\mathbb{R}} u_{x}^{2 n-1} u_{x} d x .
\end{aligned}
$$

Similarly, the following inequality holds

$$
\begin{aligned}
\frac{d}{d t}\left\|u_{x}(t)\right\|_{L^{2 n}} \leq & \left(2\left\|u_{x}(t)\right\|_{L^{\infty}}+\lambda\right)\left\|u_{x}(t)\right\|_{L^{2 n}} \\
& +\left\|\partial_{x}^{2} G * F(u)(t)\right\|_{L^{2 n}},
\end{aligned}
$$

and therefore as before, we obtain

$$
\left\|u_{x}(t)\right\|_{L^{2 n}} \leq c e^{2 M t}\left(\left\|u_{x}(0)\right\|_{L^{2 n}}+\int_{0}^{t}\left\|\partial_{x}^{2} G * F(u)\right\|_{L^{2 n}} d \tau\right) .
$$

Taking the limits in (53), we obtain

$$
\left\|u_{x}(t)\right\|_{L^{\infty}} \leq c e^{2 M t}\left(\left\|u_{x}(0)\right\|_{L^{\infty}}+\int_{0}^{t}\left\|\partial_{x}^{2} G * F(u)\right\|_{L^{\infty}} d \tau\right) .
$$

Next, we will introduce the weight function to get our desired result. This function $\varphi_{N}(x)$ with $N \in \mathbb{Z}^{+}$is independent of $t$ as the following:

$$
\varphi_{N}(x)= \begin{cases}1, & x \leq 1 \\ x^{\theta / \alpha}, & x \in(1, N) \\ N^{\theta / \alpha}, & x \geq N\end{cases}
$$

From (6) and (49), we get the following two equations:

$$
\begin{gathered}
\varphi_{N} u_{t}+\varphi_{N} u u_{x}+\varphi_{N} c_{0} u_{x}+\varphi_{N} \partial_{x} G * F(u)+\lambda \varphi_{N} u=0 \\
\varphi_{N} u_{x t}+\varphi_{N} u u_{x x}+\varphi_{N} c_{0} u_{x x}+\varphi_{N} u_{x}^{2} \\
+\varphi_{N} \partial_{x}^{2} G * F(u)+\lambda \varphi_{N} u_{x}=0 .
\end{gathered}
$$

We need some tricks to deal with the following term as in [18]:

$$
\begin{aligned}
\int_{\mathbb{R}}\left(\varphi_{N}\right)^{2 n-1} u^{2 n-1} \varphi_{N} u_{x} d x \\
\quad=\int_{\mathbb{R}}\left(\varphi_{N} u\right)^{2 n-1}\left[\left(u \varphi_{N}\right)_{x}-u\left(\varphi_{N}\right)_{x}\right] d x \\
\quad=\int_{\mathbb{R}}\left(\varphi_{N} u\right)^{2 n-1} d\left(\varphi_{N} u\right)-\int_{\mathbb{R}}\left(\varphi_{N} u\right)^{2 n-1} u\left(\varphi_{N}\right)_{x} d x \\
\quad \leq \int_{\mathbb{R}}\left(\varphi_{N} u\right)^{2 n} d x,
\end{aligned}
$$

where we have used the fact $0 \leq \varphi_{N}^{\prime}(x) \leq \varphi_{N}(x)$, a.e. $x \in \mathbb{R}$. Similar technique is used for the term $\int_{\mathbb{R}}\left(\varphi_{N}\right)^{2 n-1} u_{x}^{2 n-1} \varphi_{N} u_{x x} d x$. Hence, as in the weightless case, we get the following inequality in view of (48) and (54) as follows:

$$
\begin{aligned}
\| u(t) & \varphi_{N}\left\|_{L^{\infty}}+\right\| u_{x}(t) \varphi_{N} \|_{L^{\infty}} \\
\leq & c e^{2 M t}\left(\left\|u(0) \varphi_{N}\right\|_{L^{\infty}}+\left\|u_{x}(0) \varphi_{N}\right\|_{L^{\infty}}\right)+c e^{2 M t} \\
\quad & \times\left(\int_{0}^{t}\left(\left\|\varphi_{N} \partial_{x} G * F(u)\right\|_{L^{\infty}}+\left\|\varphi_{N} \partial_{x}^{2} G * F(u)\right\|_{L^{\infty}}\right) d \tau\right) .
\end{aligned}
$$

On the other hand, a simple calculation shows that there exists $C>0$, depending only on $\alpha$ and $\theta$ such that for any $N \in \mathbb{Z}^{+}$,

$$
\varphi_{N}(x) \int_{\mathbb{R}} e^{-|x-y| / \alpha} \frac{1}{\varphi_{N}(y)} d y \leq C .
$$


Therefore, for any appropriate function $g$, one obtains that

$$
\begin{aligned}
& \left|\varphi_{N} \partial_{x} G * g^{2}(x)\right| \\
& \quad=\left|\frac{1}{2 \alpha} \varphi_{N}(x) \int_{\mathbb{R}} e^{-|x-y| / \alpha} g^{2}(y) d y\right| \\
& \quad \leq \frac{1}{2 \alpha} \varphi_{N}(x) \int_{\mathbb{R}} e^{-|x-y| / \alpha} \frac{1}{\varphi_{N}(y)} \varphi_{N}(y) g(y) g(y) d y \\
& \quad \leq \frac{1}{2 \alpha}\left(\varphi_{N}(x) \int_{\mathbb{R}} e^{-|x-y| / \alpha} \frac{1}{\varphi_{N}(y)} d y\right)\left\|g \varphi_{N}\right\|_{L^{\infty}}\|g\|_{L^{\infty}} \\
& \leq \frac{C}{\alpha}\left\|g \varphi_{N}\right\|_{L^{\infty}}\|g\|_{L^{\infty}},
\end{aligned}
$$

and similarly, $\left|\varphi_{N} \partial_{x}^{2} G * g^{2}(x)\right| \leq(C / \alpha)\left\|g \varphi_{N}\right\|_{L^{\infty}}\|g\|_{L^{\infty}}$. Using the same method, we can estimate the following two terms

$$
\begin{gathered}
\left|\varphi_{N} G * g(x)\right| \leq \frac{C}{\alpha}\left\|g \varphi_{N}\right\|_{L^{\infty}}, \\
\left|\varphi_{N} \partial_{x} G * g(x)\right| \leq \frac{C}{\alpha}\left\|g \varphi_{N}\right\|_{L^{\infty}} .
\end{gathered}
$$

Therefore, it follows that there exists a constant $C_{1}(M, T, \alpha, \lambda)>0$ such that

$$
\begin{gathered}
\left\|u(t) \varphi_{N}\right\|_{L^{\infty}}+\left\|u_{x}(t) \varphi_{N}\right\|_{L^{\infty}} \\
\leq C_{1}\left(\left\|u(0) \varphi_{N}\right\|_{L^{\infty}}+\left\|u_{x}(0) \varphi_{N}\right\|_{L^{\infty}}\right) \\
+C_{1} \int_{0}^{t}\left(\left(\|u(\tau)\|_{L^{\infty}}+\left\|u_{x}(\tau)\right\|_{L^{\infty}}\right)\right. \\
\left.\cdot\left(\left\|\varphi_{N} u(\tau)\right\|_{L^{\infty}}+\left\|\varphi_{N} u_{x}(\tau)\right\|_{L^{\infty}}\right)\right) d \tau \\
\leq C_{1}\left(\left\|u(0) \varphi_{N}\right\|_{L^{\infty}}+\left\|u_{x}(0) \varphi_{N}\right\|_{L^{\infty}}\right. \\
\left.\quad+\int_{0}^{t}\left(\left\|\varphi_{N} u(\tau)\right\|_{L^{\infty}}+\left\|\varphi_{N} u_{x}(\tau)\right\|_{L^{\infty}}\right) d \tau\right) .
\end{gathered}
$$

Hence, the following inequality is obtained for any $N \in \mathbb{Z}^{+}$ and any $t \in[0, T]$ :

$$
\begin{aligned}
& \| u(t) \varphi_{N}\left\|_{L^{\infty}}+\right\| u_{x}(t) \varphi_{N} \|_{L^{\infty}} \\
& \leq C_{1}\left(\left\|u(0) \varphi_{N}\right\|_{L^{\infty}}+\left\|u_{x}(0) \varphi_{N}\right\|_{L^{\infty}}\right) \\
& \leq C_{1}\left(\left\|u(0) \max \left(1, x^{\theta / \alpha}\right)\right\|_{L^{\infty}}\right. \\
&\left.+\left\|u_{x}(0) \max \left(1, x^{\theta / \alpha}\right)\right\|_{L^{\infty}}\right) .
\end{aligned}
$$

Finally, taking the limit as $N$ goes to infinity in the above inequality, we can find that for any $t \in[0, T]$,

$$
\begin{gathered}
\left(\left|u(x, t) x^{\theta / \alpha}\right|+\left|u_{x}(x, t) x^{\theta / \alpha}\right|\right) \\
\leq C_{1}\left(\left\|u(0) \max \left(1, x^{\theta / \alpha}\right)\right\|_{L^{\infty}}\right. \\
\left.+\left\|u_{x}(0) \max \left(1, x^{\theta / \alpha}\right)\right\|_{L^{\infty}}\right),
\end{gathered}
$$

which completes the proof of Theorem 6 .

\section{Asymptotic Description}

The following result is to give a detailed description on the corresponding strong solution $u(x, t)$ in its lifespan with $u_{0}(x)$ being compactly supported.

Theorem 7. Assume that the initial datum $0 \not \equiv u_{0}(x) \in$ $H^{s}$ with $s>5 / 2$ is compactly supported in $[a, c]$, then the corresponding solution $u(x, t) \in C\left([0, T) ; H^{s}\right)$ to $(6)$ has the following property: for any $t \in(0, T)$,

$$
\begin{gathered}
u(x, t)=L(t) e^{-x / \alpha} \quad \text { as } x>q(c, t), \\
u(x, t)=l(t) e^{x / \alpha} \quad \text { as } x<q(a, t),
\end{gathered}
$$

where $q(x, t)$ is defined by (24) and $T$ is its lifespan. Furthermore, $L(t)$ and $l(t)$ denote continuous nonvanishing functions, with $L(t)>0$ and $l(t)<0$ for $t \in(0, T)$. Moreover, $L(t)$ is a strictly increasing function, while $l(t)$ is strictly decreasing.

Remark 8. This is an interesting phenomenon for our model; it implies that the strong solution does not have compact $x$ support for any $t>0$ in its lifespan anymore, although the corresponding $u_{0}(x)$ is compactly supported. No matter that the initial profile $u_{0}(x)$ is (no matter it is positive or negative), for any $t>0$ in its lifespan, the nontrivial solution $u(x ; t)$ is always positive at infinity and negative at negative infinity. Moreover, we found that the dissipative coefficient does not affect this behavior.

Proof. First, since $u_{0}(x)$ has a compact support, so does $y_{0}(x)=\left(1-\alpha^{2} \partial_{x}^{2}\right) u_{0}(x)$. Equation (30) tells us that $y=$ $\left(1-\alpha^{2} \partial_{x}^{2}\right) u(x, t)=\left(\left(1-\alpha^{2} \partial_{x}^{2}\right) u_{0}\left(q^{-1}(x, t)\right) \exp (-\lambda t)\right) /$ $\left(\partial_{x} q^{-1}((x, t), t)\right)^{2}$ is compactly supported in $[q(a, t), q(c, t)]$ in its lifespan. Hence, the following functions are well defined

$$
E(t)=\int_{\mathbb{R}} e^{\xi / \alpha} y(\xi, t) d \xi, \quad F(t)=\int_{\mathbb{R}} e^{-\xi / \alpha} y(\xi, t) d \xi,
$$

with

$$
\begin{aligned}
E(0) & =\int_{\mathbb{R}} e^{\xi / \alpha} y_{0}(\xi) d \xi \\
& =\int_{\mathbb{R}} e^{\xi / \alpha} u_{0}(\xi) d \xi-\alpha^{2} \int_{\mathbb{R}} e^{\xi / \alpha} u_{0 x x}(\xi) d \xi=0 .
\end{aligned}
$$

And $F(0)=0$ by integration by parts.

Then, for $x>q(c, t)$, we have

$$
\begin{aligned}
u(x, t) & =\frac{1}{2 \alpha} e^{-|x| / \alpha} * y(x, t) \\
& =\frac{1}{2 \alpha} e^{-x / \alpha} \int_{q(a, t)}^{q(c, t)} e^{\xi / \alpha} y(\xi, t) d \xi=\frac{1}{2 \alpha} e^{-x / \alpha} E(t),
\end{aligned}
$$

where (66) is used. 
Similarly, when $x<q(a, t)$, we get

$$
\begin{aligned}
u(x, t) & =\frac{1}{2 \alpha} e^{-|x| / \alpha} * y(x, t) \\
& =\frac{1}{2 \alpha} e^{x / \alpha} \int_{q(a, t)}^{q(c, t)} e^{-\xi / \alpha} y(\xi, t) d \xi=\frac{1}{2 \alpha} e^{x / \alpha} F(t) .
\end{aligned}
$$

Because $y(x, t)$ has a compact support in $x$ in the interval $[q(a, t), q(c, t)]$ for any $t \in[0, T]$, we get $y(x, t)=u(x, t)-$ $\alpha^{2} u_{x x}(x, t)=0$, for $x>q(c, t)$ or $x<q(a, t)$. Hence, as consequences of (68) and (69), we have

$$
\begin{aligned}
u(x, t) & =-\alpha u_{x}(x, t)=\alpha^{2} u_{x x}(x, t) \\
& =\frac{1}{2 \alpha} e^{-x / \alpha} E(t), \quad \text { as } x>q(c, t), \\
u(x, t) & =\alpha u_{x}(x, t)=\alpha^{2} u_{x x}(x, t) \\
& =\frac{1}{2 \alpha} e^{x / \alpha} F(t), \quad \text { as } x<q(a, t) .
\end{aligned}
$$

On the other hand,

$$
\frac{d E(t)}{d t}=\int_{\mathbb{R}} e^{\xi / \alpha} y_{t}(\xi, t) d x
$$

Substituting the identity (10) into $d E(t) / d t$, we obtain

$$
\begin{aligned}
\frac{d E(t)}{d t}= & -\int_{\mathbb{R}} e^{\xi / \alpha}\left[(y u)_{x}+\frac{1}{2}\left(u^{2}-\alpha^{2} u_{x}^{2}\right)_{x}+c_{0} y_{x}+\lambda y\right] d \xi \\
= & \frac{1}{\alpha} \int_{\mathbb{R}} e^{\xi / \alpha} y u d \xi+\frac{1}{2 \alpha} \int_{\mathbb{R}} e^{\xi / \alpha}\left(u^{2}-\alpha^{2} u_{x}^{2}\right) d \xi \\
& +\frac{c_{0}}{\alpha} \int_{\mathbb{R}} e^{\xi / \alpha} y d \xi+\alpha^{2} \int_{\mathbb{R}} e^{\xi / \alpha} \lambda u_{x x} d \xi \\
& -\int_{\mathbb{R}} e^{\xi / \alpha} \lambda u d \xi=\frac{3}{2 \alpha} \int_{\mathbb{R}} e^{\xi / \alpha} u^{2} d \xi+\frac{\alpha}{2} \int_{\mathbb{R}} e^{\xi / \alpha} u_{x}^{2} d \xi \\
& +\int_{\mathbb{R}} e^{\xi / \alpha} u u_{x} d \xi=\int_{\mathbb{R}} e^{\xi / \alpha}\left(\frac{1}{\alpha} u^{2}+\frac{\alpha}{2} u_{x}^{2}\right) d \xi>0,
\end{aligned}
$$

where we used (70). Therefore, in the lifespan of the solution, we have that $E(t)$ is an increasing function with $E(0)=0$; thus, it follows that $E(t)>0$ for $t \in(0, T]$; that is,

$$
E(t)=\int_{0}^{t} \int_{\mathbb{R}} e^{\xi / \alpha}\left(\frac{1}{\alpha} u^{2}+\frac{\alpha}{2} u_{x}^{2}\right)(\xi, \tau) d \xi d \tau>0
$$

By similar argument, one can verify that the following identity for $F(t)$ is true:

$$
F(t)=-\int_{0}^{t} \int_{\mathbb{R}} e^{-\xi / \alpha}\left(\frac{1}{\alpha} u^{2}+\frac{\alpha}{2} u_{x}^{2}\right)(\xi, \tau) d \xi d \tau<0 .
$$

In order to finish the proof, it is sufficient to let $L(t)=$ $(1 / 2 \alpha) E(t)$, and to let $l(t)=(1 / 2 \alpha) F(t)$, respectively.

\section{References}

[1] H. R. Dullin, G. A. Gottwald, and D. D. Holm, "An integrable shallow water equation with linear and nonlinear dispersion," Physical Review Letters, vol. 87, no. 19, Article ID 194501, 4 pages, 2001.

[2] J. Bourgain, "Fourier transform restriction phenomena for certain lattice subsets and applications to nonlinear evolution equations, II. The KdV equation," Geometric and Functional Analysis, vol. 3, no. 3, pp. 209-262, 1993.

[3] R. Camassa and D. D. Holm, "An integrable shallow water equation with peaked solitons," Physical Review Letters, vol. 71, no. 11, pp. 1661-1664, 1993.

[4] A. Constantin, "Finite propagation speed for the CamassaHolm equation," Journal of Mathematical Physics, vol. 46, no. 2, Article ID 023506, 4 pages, 2005.

[5] A. Constantin and J. Escher, "Wave breaking for nonlinear nonlocal shallow water equations," Acta Mathematica, vol. 181, no. 2, pp. 229-243, 1998.

[6] A. Constantin and J. Escher, "Well-posedness, global existence, and blowup phenomena for a periodic quasi-linear hyperbolic equation," Communications on Pure and Applied Mathematics, vol. 51, no. 5, pp. 475-504, 1998.

[7] A. A. Himonas, G. Misiołek, G. Ponce, and Y. Zhou, "Persistence properties and unique continuation of solutions of the Camassa-Holm equation," Communications in Mathematical Physics, vol. 271, no. 2, pp. 511-522, 2007.

[8] H. P. McKean, "Breakdown of the Camassa-Holm equation," Communications on Pure and Applied Mathematics, vol. 57, no. 3, pp. 416-418, 2004.

[9] Z. Xin and P. Zhang, "On the weak solutions to a shallow water equation," Communications on Pure and Applied Mathematics, vol. 53, no. 11, pp. 1411-1433, 2000.

[10] Y. Zhou, "Wave breaking for a shallow water equation," Nonlinear Analysis: Theory, Methods \& Application, vol. 57, no. 1, pp. 137-152, 2004.

[11] Y. Zhou, "Wave breaking for a periodic shallow water equation," Journal of Mathematical Analysis and Applications, vol. 290, no. 2, pp. 591-604, 2004.

[12] Y. Zhou, "Stability of solitary waves for a rod equation," Chaos, Solitons and Fractals, vol. 21, no. 4, pp. 977-981, 2004.

[13] Y. Zhou, "Local well-posedness and blow-up criteria of solutions for a rod equation," Mathematische Nachrichten, vol. 278, no. 14, pp. 1726-1739, 2005.

[14] L. Ni and Y. Zhou, "Wave breaking and propagation speed for a class of nonlocal dispersive $\theta$-equations," Nonlinear Analysis: Real World Applications, vol. 12, no. 1, pp. 592-600, 2011.

[15] L. Tian, G. Gui, and Y. Liu, "On the well-posedness problem and the scattering problem for the Dullin-Gottwald-Holm equation," Communications in Mathematical Physics, vol. 257, no. 3, pp. 667-701, 2005.

[16] T. Kato, "Quasi-linear equations of evolution, with applications to partial differential equations," in Spectral Theory and Differential Equations: Proceedings of the Symposium Held at Dundee, Scotland, 1-19 July 1974, vol. 448 of Lecture Notes in Mathetics, pp. 25-70, Springer, Berlin, Germany, 1975.

[17] Y. Zhou, "Blow-up of solutions to the DGH equation," Journal of Functional Analysis, vol. 250, no. 1, pp. 227-248, 2007.

[18] Y. Zhou and Z. Guo, "Blow up and propagation speed of solutions to the DGH equation," Discrete and Continuous Dynamical Systems B, vol. 12, no. 3, pp. 657-670, 2009. 
[19] J.-M. Ghidaglia, "Weakly damped forced Korteweg-de Vries equations behave as a finite-dimensional dynamical system in the long time," Journal of Differential Equations, vol. 74, no. 2, pp. 369-390, 1988.

[20] Z. Guo, "Blow up, global existence, and infinite propagation speed for the weakly dissipative Camassa-Holm equation," Journal of Mathematical Physics, vol. 49, no. 3, Article ID 033516, 9 pages, 2008.

[21] Z. Guo and L. Ni, "Wave breaking for the periodic weakly dissipative Dullin-Gottwald-Holm equation," Nonlinear Analysis: Theory, Methods \& Applications, vol. 74, no. 3, pp. 965-973, 2011.

[22] S. Wu and Z. Yin, "Global existence and blow-up phenomena for the weakly dissipative Camassa-Holm equation," Journal of Differential Equations, vol. 246, no. 11, pp. 4309-4321, 2009.

[23] A. V. Busuioc and T. S. Ratiu, "The second grade fluid and averaged Euler equations with Navier-slip boundary conditions," Nonlinearity, vol. 16, no. 3, pp. 1119-1149, 2003. 


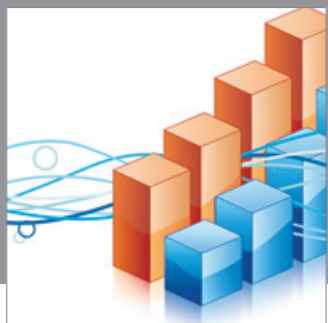

Advances in

Operations Research

mansans

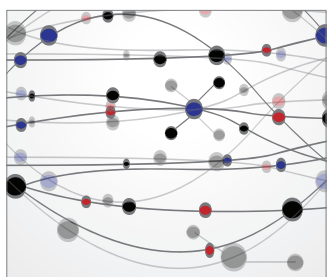

The Scientific World Journal
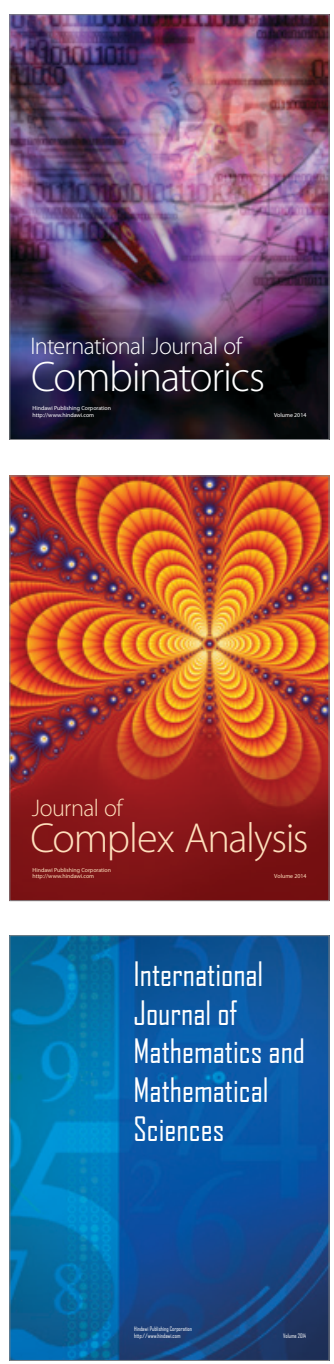
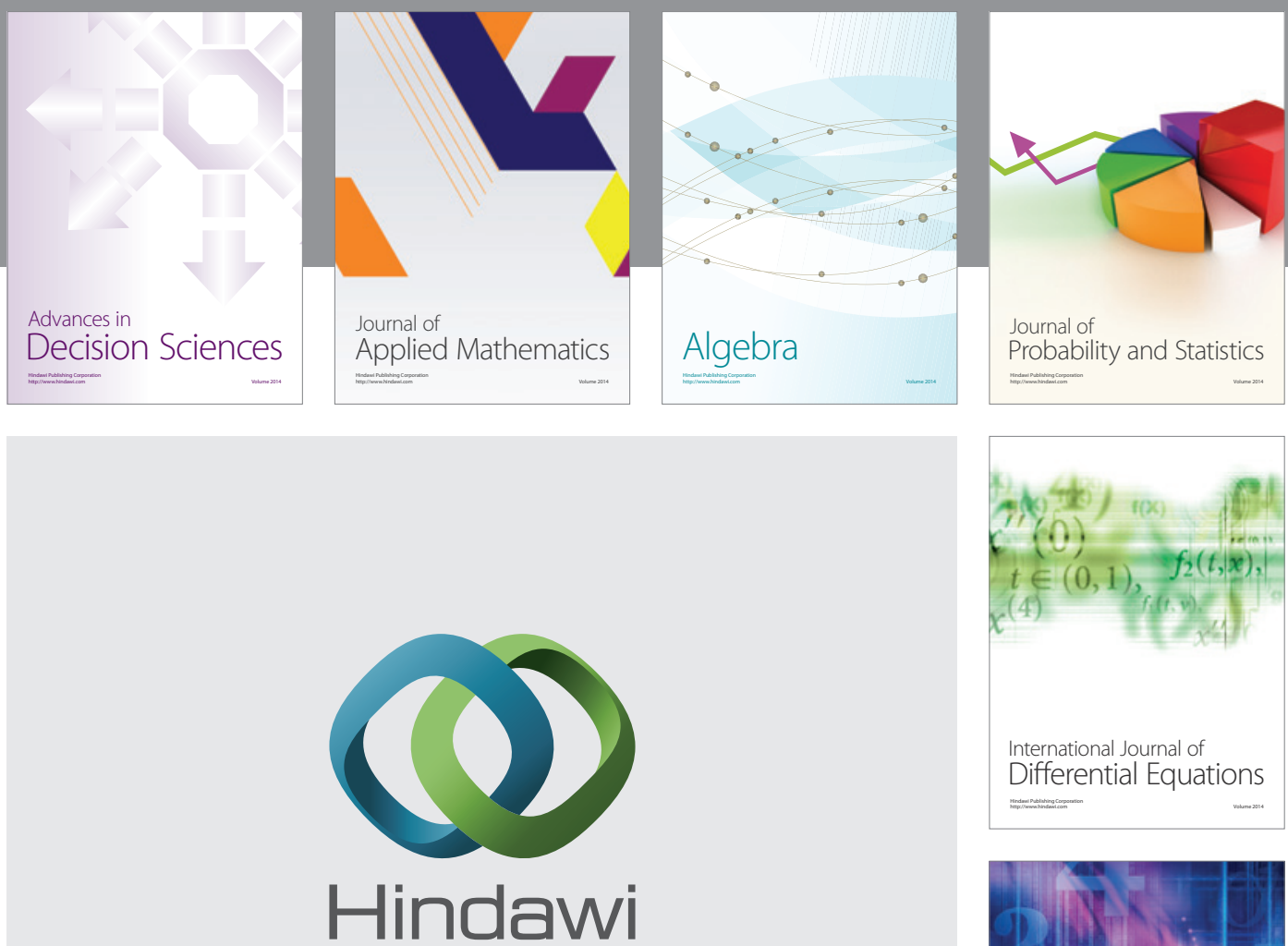

Submit your manuscripts at http://www.hindawi.com
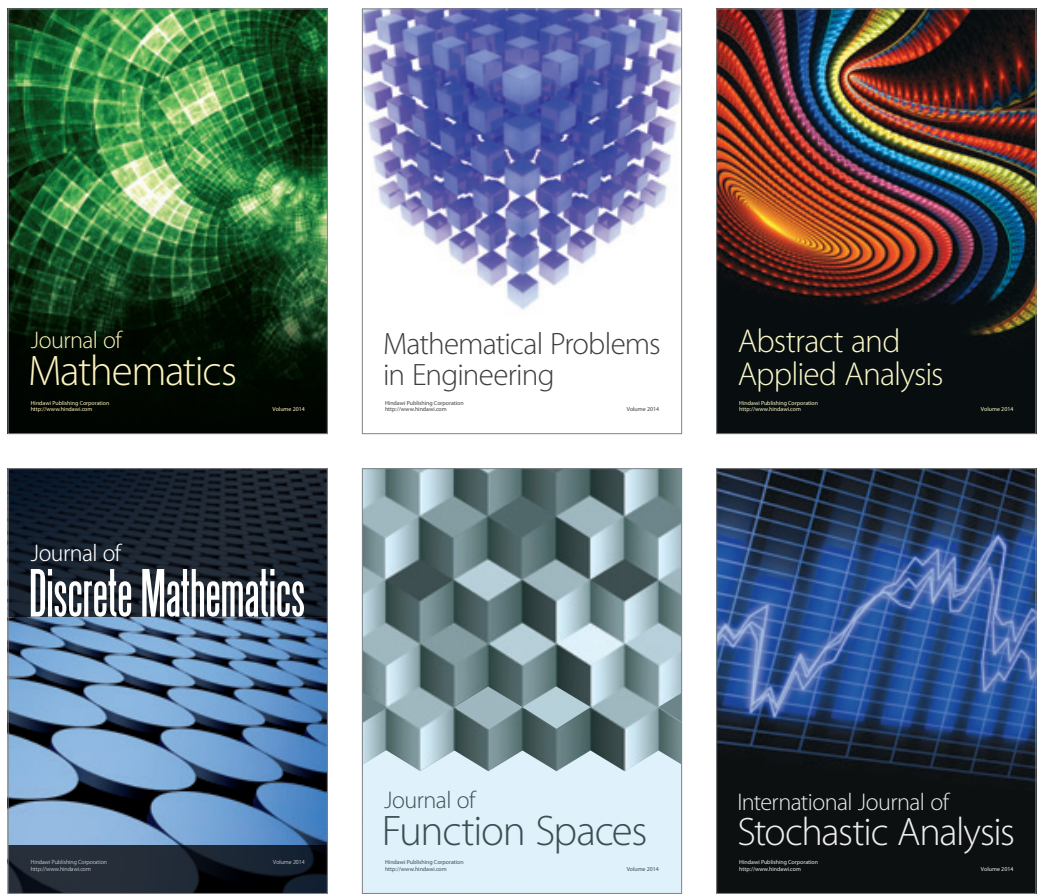

Journal of

Function Spaces

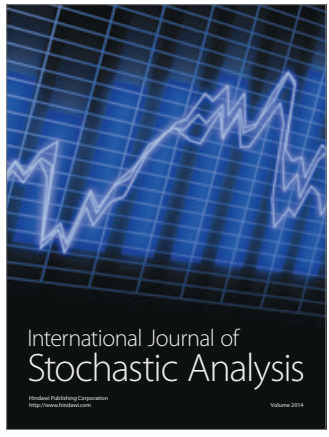

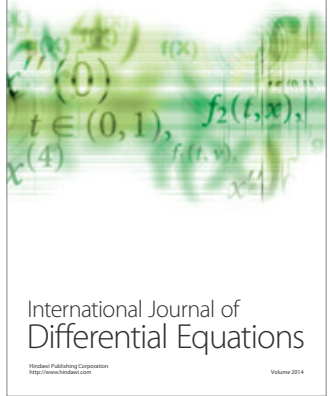
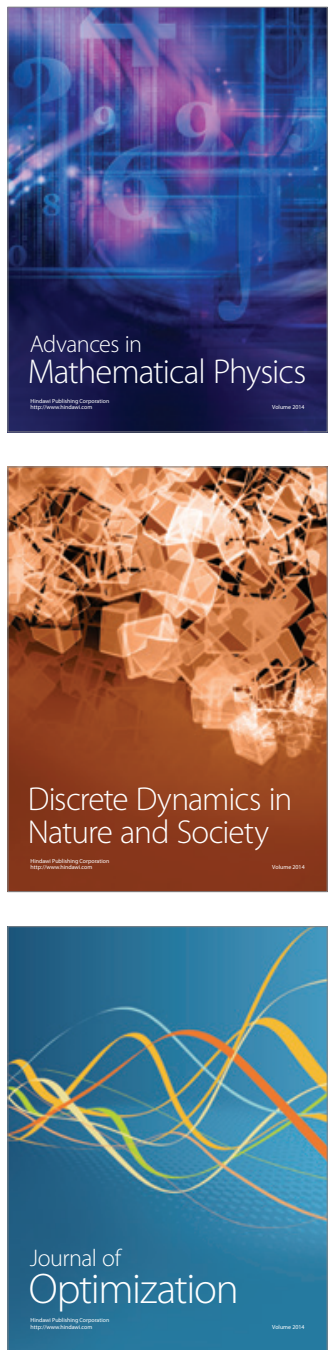